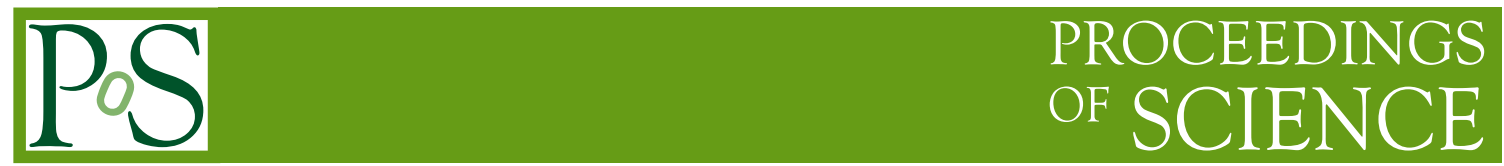

\title{
Measurement of semitauonic b-hadron decays
}

\author{
Biljana Mitreska ${ }^{a, 1, *}$ \\ ${ }^{a}$ The University of Manchester, \\ Department of Physics and Astronomy, Manchester, UK \\ E-mail: biljana.mitreska@cern.ch
}

The semileptonic b-hadron decays with a heavy lepton are sensitive to new couplings like those generated by charged Higgs or Leptoquarks. The B-Factories and LHCb have previously performed various measurements of these decays, using different approaches and techniques. A global average of these measurements shows a discrepancy with the Standard Model expectations, which is above 3 standard deviations.

The European Physical Society Conference on High Energy Physics (EPS-HEP2021), 26-30 July 2021

Online conference, jointly organized by Universität Hamburg and the research center DESY

\footnotetext{
${ }^{1}$ On behalf of the LHCb collaboration

* Speaker
} 


\section{Introduction}

In the Standard Model (SM) of Particle Physics the couplings of the gauge bosons are independent of the lepton flavour, also known as Lepton Flavour Universality (LFU). If any violation of LFU is measured it leads to a clear sign of beyond SM (BSM) physics. The semileptonic b-hadron decays have a simple SM description with a tree level diagram and high branching fractions. They are powerful probes for testing the SM and performing searches for BSM effects. To test any discrepancies between decays to different leptons the ratio of branching fractions of b-hadrons having a $\tau$ and a $\mu$ in their final states is used, defined as

$$
\mathcal{R}\left(H_{c}\right)=\frac{\mathcal{B}\left(H_{b} \rightarrow H_{c} \tau v\right)}{\mathcal{B}\left(H_{b} \rightarrow H_{c} \mu \nu\right)}
$$

where $H_{c}=D^{*}, D^{+}, J / \psi, \ldots$ and $H_{b}=B^{ \pm}, B^{0}$. The combined measurements of the ratios $\mathcal{R}(D)$ and $\mathcal{R}\left(D^{*}\right)$ have been performed at Belle [1] [2] [3] [4] and BaBar [5] [6] and the ratio $\mathcal{R}\left(D^{*}\right)$ has been measured by LHCb [7] [8]. These measurements have an overall tension of slightly above 3.0 $\sigma$ with the SM prediction [9] [10] [11], shown on Fig. 1.

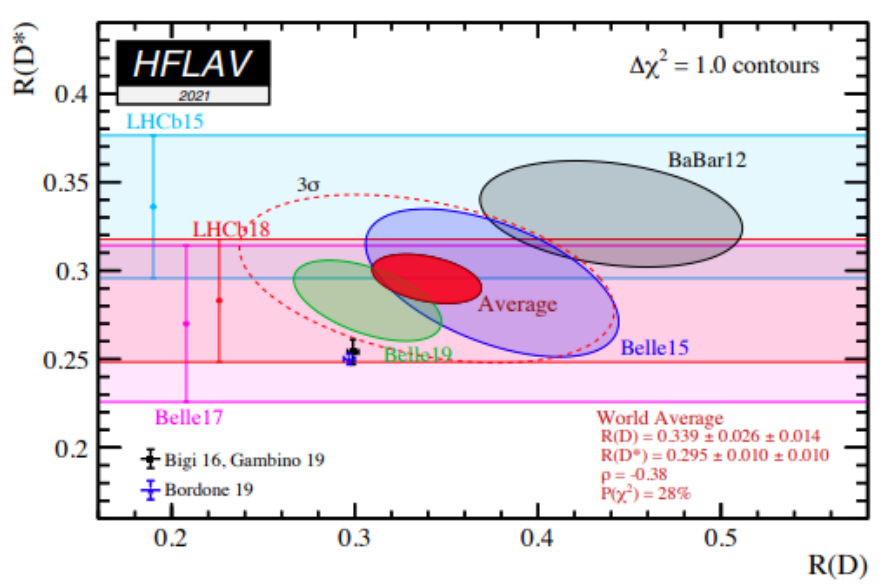

Figure 1: Summary of measurements of $\mathcal{R}(D)$ and $\mathcal{R}\left(D^{*}\right)$ by the Heavy Flavor Averaging Group [12]. The average of the experimental results is shown as a red ellipse and the dotted lines indicate the $3 \sigma$ region. The black and blue points show the SM prediction.

The LHCb experiment contributed to this combination with two measurements of $\mathcal{R}\left(D^{*}\right)$ using the Run 1 dataset with a centre-of-mass energy of 7 and $8 \mathrm{TeV}$ corresponding to $3 \mathrm{fb}^{-1}$ integrated luminosity. These measurements are performed using two different $\tau$ decays: $\tau \rightarrow \mu v v$ and $\tau \rightarrow \pi \pi \pi v$. The inability of LHCb to detect neutrinos is a challenge for all semileptonic measurements performed there. Therefore specific procedures need to be followed estimate the kinematic properties of the decay. 


\section{LHCb measurements}

\subsection{Measurement of $\mathcal{R}\left(D^{*}\right)$ with $\tau \rightarrow \mu \nu v$}

This measurement proceeds with the muonic decay of the $\tau$ and having neutrinos present in the signal and normalisation channel prevents the full reconstruction of the decay. An approximation for the B momentum is derived in which the B rest frame is used assuming that the proper velocity of the $\mathrm{B}$ meson along the beam axis is the same as the velocity of the $\mathrm{D}^{*} \mu$ system (the visible part of the decay). For the main backgrounds a Boosted Decision Tree (BDT) that is made to isolate the signal and reject backgrounds with additional charged tracks is used. The BDT uses track and $\mathrm{B}$ vertex properties and determines if a given track originates from the same B candidate or from anywhere else in the rest of the event. The final fit strategy consists of a binned fit in the muon energy in the estimated $\mathrm{B}$ rest frame $\left(E_{\mu}\right)$, the missing mass $\left(m_{m i s s}^{2}=\left(p_{B}-p_{D^{*}}-p_{l}\right)^{2}\right)$ and the momentum transfer $\left(q^{2}=\left(p_{B}-p_{D^{*}}\right)^{2}\right)$, where $p_{B}, p_{D^{*}}$ and $p_{l}$ are the momenta of the $\mathrm{B}, \mathrm{D}^{*}$ and the lepton. The final result is $R\left(D^{*}\right)=0.336 \pm 0.027$ (stat) \pm 0.030 (syst) with the main systematic uncertainty due to the size of the Monte Carlo samples and the backgrounds from hadrons misidentified as muons. The final fit projections are shown on Fig. 2 [7]. This result is 2.1 $\sigma$ greater than the SM expectation.
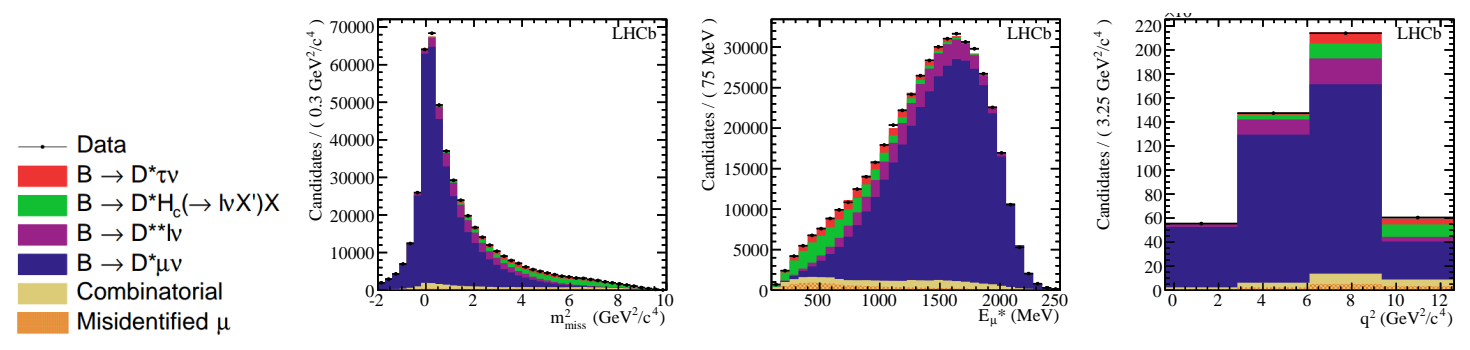

Figure 2: Fit projections of distributions of $m_{m i s s}^{2}, E_{\mu}$ and $q^{2}$ for Run $1 R\left(D^{*}\right)$ measurement with $\tau \rightarrow \mu v v$ [7].

\subsection{Measurement of $\mathcal{R}\left(D^{*}\right)$ with $\tau \rightarrow \pi \pi \pi v$}

This $\mathcal{R}\left(D^{*}\right)$ measurement uses hadronic $\tau$ final state for the $\tau$ decay mode. In order to retain the same final state as the normalisation the quantity $\kappa\left(D^{*}\right)=\frac{\mathcal{B}\left(B \rightarrow D^{*} \tau \nu\right)}{\mathcal{B}\left(B \rightarrow D^{*-3 \pi)}\right.}$ is defined. Using this the ratio is derived as

$$
\mathcal{R}\left(D^{*}\right)=\kappa\left(D^{*}\right) \frac{\mathcal{B}\left(B \rightarrow D^{*} 3 \pi\right)}{\mathcal{B}\left(B \rightarrow D^{*} \mu \nu\right)},
$$

where an external input is taken for $\mathcal{B}\left(B \rightarrow D^{*} 3 \pi\right)$ and $\mathcal{B}\left(B \rightarrow D^{*} \mu v\right)$. A correction for the missing neutrino on the $\mathrm{B}$ and $\tau$ momentum is applied using the two solutions approach [13]. One of the main backgrounds coming from $B \rightarrow D^{*-} 3 \pi X$ is suppressed via the $3 \pi$ vertex requirement which uses the ability to reconstruct the detached $\tau(3 \pi)$ vertex. For the suppression of the double charm backgrounds $\left(B \rightarrow D^{*-} D_{s}^{+} X, B \rightarrow D^{*-} D^{+} X, B \rightarrow D^{*-} D^{0} X\right)$ a BDT is applied. The normalisation channel yield is extracted from an unbinned maximum likelihood fit to $M\left(D^{*-} \pi^{-} \pi^{+} \pi^{-}\right)$and the signal extraction proceeds with a binned fit in $q^{2}, \tau$ decay time and the BDT output. The result obtained is $\mathcal{R}\left(D^{*}\right)=0.280 \pm 0.018$ (stat) \pm 0.029 (syst) with dominant systematic uncertainty 
from the modelling of different backgrounds, form factors and the statistics of templates [8]. This measurement reports a value that is $1 \sigma$ higher than the SM prediction.

\subsection{Measurement of $\mathcal{R}(J / \psi)$ with $\tau \rightarrow \mu v v$}

The $\mathcal{R}(J / \psi)$ ratio measured by LHCb is defined as $\mathcal{R}(J / \psi)=\frac{\mathcal{B}\left(B_{c} \rightarrow J / \psi \tau \nu\right)}{\mathcal{B}\left(B_{c} \rightarrow J / \psi \mu \nu\right)}$ being the first study of $B_{c} \rightarrow J / \psi \tau v$ from LHCb. The signal and normalisation extraction uses a 3D binned fit in the $\mathrm{B}_{\mathrm{c}}$ lifetime, $m_{\text {miss }}^{2}$ and a variable $Z$ derived from $q^{2}$ and $E_{\mu}$. The measured result is $\mathcal{R}(J / \psi)=0.71 \pm 0.17$ (stat) \pm 0.18 (syst) with the main systematics coming from the poor knowledge of the $\mathrm{B}_{\mathrm{c}}$ form factors [14]. Fig. 3 shows the final projections of the fit variables with the result being $2 \sigma$ away from the SM prediction.
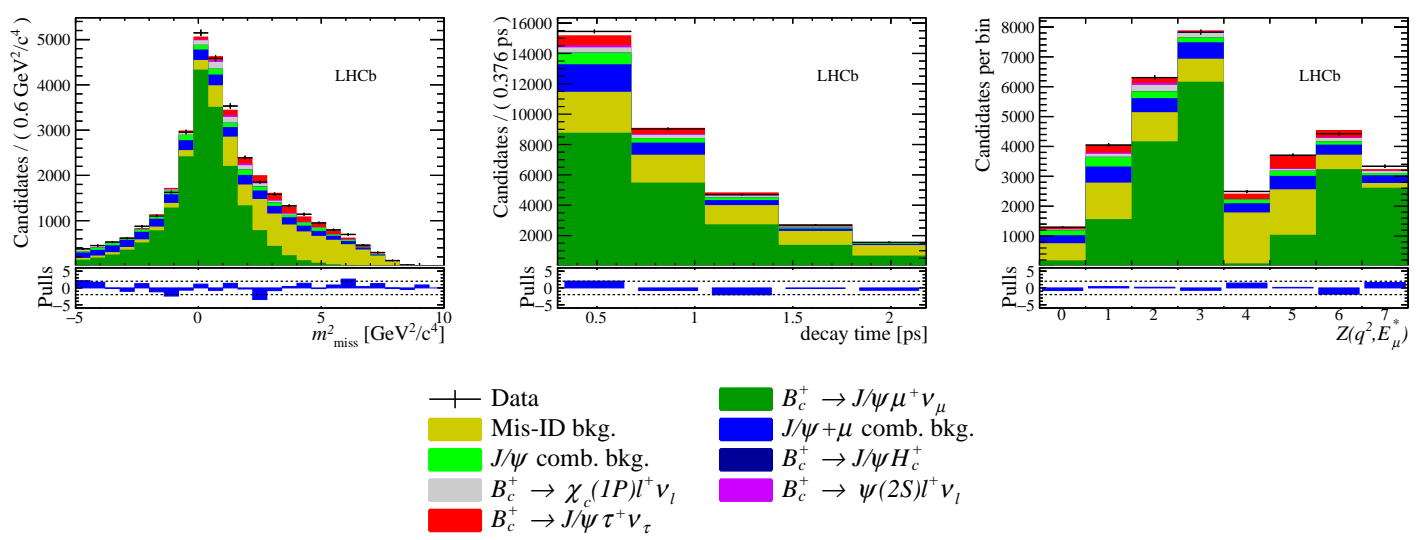

Figure 3: Fit projections of distributions of $m_{m i s s}^{2}$, decay time and the variable $Z$ (derived from $E_{\mu}$ and $q^{2}$ ) [14].

\section{Future prospects}

LHCb is continuing to test the LFU hypothesis in a range of new analyses using $b \rightarrow c l v$ decays in both the muonic and hadronic $\tau$ decay channel. This includes the measurement of the combined ratios $\mathcal{B}(B \rightarrow D \tau v) / \mathcal{B}(B \rightarrow D \mu v)$ and $\mathcal{B}\left(B \rightarrow D^{*} \tau v\right) / \mathcal{B}\left(B \rightarrow D^{*} \mu v\right)$ using Run 1 LHCb data and update of the above discussed measurements with Run 2 LHCb data. Measurements such as $\mathcal{R}\left(D^{* *}\right), \mathcal{R}\left(D_{s}^{*}\right), \mathcal{R}\left(D^{*}\right)$ (electron-muon) and $\mathcal{R}\left(\Lambda_{c}^{*}\right)$ are also in progress. A complementary measurement to the ratio of branching fractions that can help to further constrain the LFU anomaly is performing an angular analysis. There are many theoretical BSM predictions [15] [16] [17] [18] and in this direction LHCb is working on several angular analyses in $B \rightarrow D^{*} \tau v$ and $B \rightarrow D^{*} \mu \nu$. Exploiting the angular structure of these decays can experimentally confirm the BSM scenarios with a great precision and further constrain the $\mathcal{R}\left(D^{*}\right)$ discrepancies. In addition, with the estimation of the BSM sensitivities in $\Lambda_{b} \rightarrow \Lambda_{c} l v$ decays [19] an angular analysis is ideally placed to confirm the presence of BSM physics there. With the Run 3 data-taking period from $2022 \mathrm{LHCb}$ will accumulate larger sample size which will help in reducing the systematic uncertainties of these measurements. The expected precision on these measurements over the years is shown on Fig. 4 with an estimation of less than $2 \%$ on the systematics on the ratios of branching fractions [20] 
[21] if in the years to come all the different challenges in computation, background modeling and estimation are met.

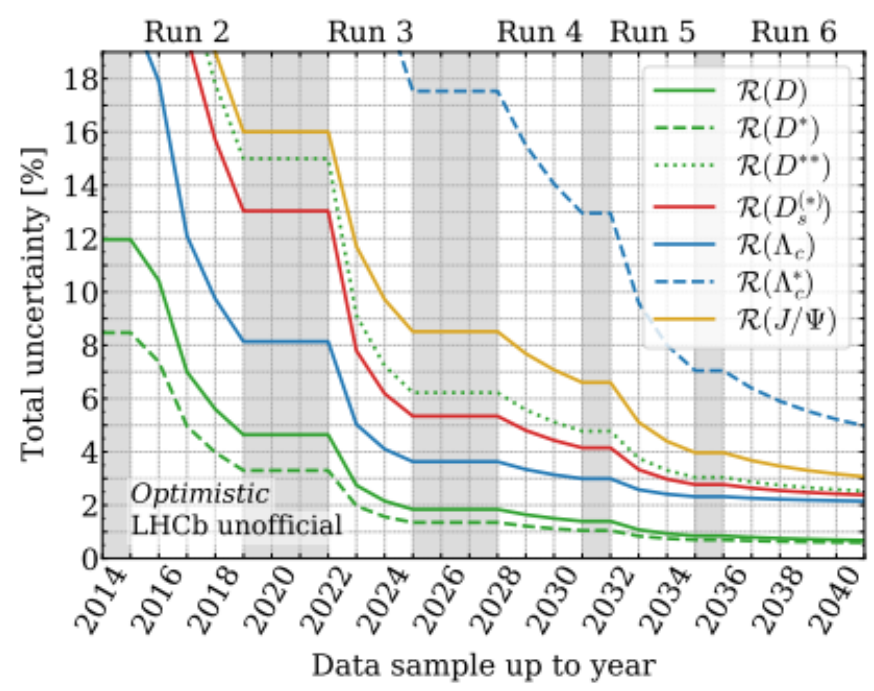

Figure 4: Projections for the expected precision on the measurement of selected $\mathcal{R}\left(H_{c}\right)$ ratios at $\mathrm{LHCb}$ as a function of the year in which the corresponding data sample becomes available [20].

\section{Conclusions}

Semileptonic decays can give important information on BSM effects. Having the $3 \sigma$ tension present in the ratio of branching fractions of b-hadron decays to $\tau$ and $\mu$ the LFU is still an ongoing puzzle. LHCb is testing the LFU by looking at the ratios of branching fractions and by performing angular analyses in $B \rightarrow D^{*} l v$ and $\Lambda_{b} \rightarrow \Lambda_{c} l v$. The data collected by LHCb during Run 3 can help to reduce the systematic uncertainties and could be sufficient to resolve the present anomalies or establish an observation of LFU violation.

\section{References}

[1] Belle collaboration, Measurement of the branching ratio of $\bar{B} \rightarrow D^{(*)} \tau^{-} \bar{v}_{\tau}$ relative to $\bar{B} \rightarrow$ $D^{(*)} \ell^{-} \bar{v}_{\ell}$ decays with hadronic tagging at Belle, Phys. Rev. D 92 (2015) 072014.

[2] Belle collaboration, Measurement of the branching ratio of $\bar{B}^{0} \rightarrow D^{*+} \tau^{-} \bar{v}_{\tau}$ relative to $\bar{B}^{0} \rightarrow$ $D^{*+} \ell^{-} \bar{v}_{\ell}$ decays with a semileptonic tagging method, Phys. Rev. D 94 (2016) 072007.

[3] Belle collaboration, Measurement of the $\tau$ lepton polarization and $R\left(D^{*}\right)$ in the decay $\bar{B} \rightarrow$ $D^{*} \tau^{-} \bar{v}_{\tau}$, Phys. Rev. Lett. 118 (2017) 211801.

[4] Belle collaboration, Measurement of $\mathcal{R}(D)$ and $\mathcal{R}\left(D^{*}\right)$ with a semileptonic tagging method, Phys. Rev. Lett. 124 (2020) 161803.

[5] BaBar collaboration, Evidence for an Excess of $\bar{B} \rightarrow D^{(*)} \tau^{-} \bar{v}_{\tau}$ Decays, Phys. Rev. Lett. 109 (2012) 101802. 
[6] BaBar collaboration, Measurement of an excess of $\bar{B} \rightarrow D^{(*)} \tau^{-} \bar{v}_{\tau}$ decays and implications for charged Higgs bosons, Phys. Rev. D 88 (2013) 072012.

[7] LHCb collaboration, Measurement of the ratio of branching fractions $\mathcal{B}\left(\bar{B}^{0} \rightarrow D^{*+} \tau^{-} \bar{v}_{\tau}\right) /$ $\mathcal{B}\left(\bar{B}^{0} \rightarrow D^{*+} \mu^{-} \bar{v}_{\mu}\right)$, Phys. Rev. Lett. 115 (2015) 111803.

[8] LHCb collaboration, Measurement of the ratio of the $B^{0} \rightarrow D^{*-} \tau^{+} v_{\tau}$ and $B^{0} \rightarrow D^{*-} \mu^{+} v_{\mu}$ branching fractions using three-prong $\tau$-Lepton decays, Phys. Rev. Lett. 120 (2018) 171802.

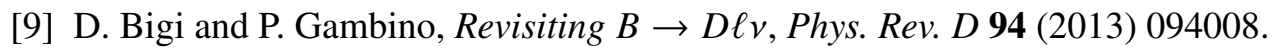

[10] P. Gambino, M. Jung and S. Schacht, The Vcb puzzle: An update, Phys. Lett. B 795 (2019) 386.

[11] M. Bordone, M. Jung and D. van Dyk, Theory determination of $\bar{B} \rightarrow D^{(*)} \ell^{-} \bar{v}$ form factors at $O\left(1 / m_{c}^{2}\right)$, Eur. Phys. J. C 80 (2020) 74.

[12] Y. Amhis et al., Averages of b-hadron, c-hadron, and tau-lepton properties as of summer 2018, Eur. Phys. J. C81 (2021) 226.

[13] LHCb collaboration, Test of lepton flavor universality by the measurement of the $B^{0} \rightarrow$ $D^{*-} \tau^{+} v_{\tau}$ branching fraction using three-prong $\tau$ decays, Phys. Rev. D 97 (2018) 072013.

[14] LHCb collaboration, Measurement of the ratio of branching fractions $B_{c}^{+} \rightarrow J / \psi \tau^{+} v_{\tau}$ and $B_{c}^{+} \rightarrow J / \psi \mu^{+} v_{\mu}$, Phys. Rev. Lett. 120 (2018) 121801.

[15] B. Dumont, K. Nishiwaki, and R. Watanabe, LHC constraints and prospects for $S_{1}$ scalar leptoquark explaining the $\bar{B} \rightarrow D^{*} \tau \bar{v}$ anomaly, Phys. Rev. D 94 (2016) 034001.

[16] M. Duraisamy, P. Sharma, and A. Datta, Azimuthal $B \rightarrow D^{*} \tau^{-} \overline{v_{\tau}}$ angular distribution with tensor operators, Phys. Rev. D 90 (2014) 074013.

[17] X. Gang He and G. Valencia, B decays with $\tau$ leptons in nonuniversal left-right models, Phys. Rev. D 87 (2013) 014014.

[18] S. Fajfer, J. F. Kamenik and I. Nisandzic, On the $B \rightarrow D^{*} \tau v_{t} \bar{a} u$ sensitivity, Phys. Rev. D 85 (2012) 094025.

[19] M. Ferrillo, A. Mathad, P. Owen and N. Serra, Probing effects of new physics in $\Lambda_{b}^{0} \rightarrow \Lambda_{c}^{+} \mu^{-} \overline{v_{\mu}}$ decays, JHEP 148 (2019).

[20] F.U Bernlochner, M. Franco Sevilla, D. J. Robinson, G. Wormser, Semitauonic b-hadron decays: A lepton flavor universality laboratory, arXiv:2101.08326v1 (2021).

[21] LHCb collaboration, Physics case for an LHCb Upgrade II, arXiv:1808.08865v4 (2019). 